\title{
ANÁLISIS DE LAS FOTOGRAFÍAS Y VÍDEOS DE INSTAGRAM PARA LA CREACIÓN DE UN RANKING DE POPULARIDAD DE LOS TERRITORIOS Y LOS DESTINOS
}

\author{
Assumpció Huertas* \\ Universitat Rovira i Virgili. Tarragona \\ https://orcid.org/0000-0001-6684-4220 \\ Xabier Martínez-Rolán** \\ Universidade de Vigo \\ https://orcid.org/0000-0002-7631-2292
}

\section{RESUMEN}

Instagram permite a los usuarios compartir fotografías y vídeos y es una herramienta clave para crear imagen de los destinos. Actualmente los destinos compiten entre sí para tener una buena imagen y un buen posicionamiento. Por ello, los objetivos de este estudio son: analizar las publicaciones georreferenciadas de fotografías y vídeos de los usuarios de destinos, las reacciones que estas han comportado y crear un Factor de Impacto que genere un ranking de popularidad de los destinos entre los usuarios en Instagram. El estudio muestra las aportaciones del Factor de Impacto en Instagram creado para medir la popularidad de los destinos respecto a rankings anteriores y muestra también su utilidad para las Organizaciones de Marketing de los Destinos.

Palabras clave: Instagram; georreferenciación, fotografías; vídeos; ranking; popularidad; destinos.

\section{Analysis of photographs and videos of Instagram for the creation of a popularity ran-} king of territories and destinations

Fecha de recepción: 29 de noviembre de 2018.

Fecha de aceptación: 26 de abril de 2019.

* Departamento de Estudios de Comunicación. Universitat Rovira i Virgili. Av. Catalunya, 35. 43002 TARRAGONA (España).E-mail: sunsi.huertas@urv.cat

** Departamento de Comunicación Audiovisual. Universidade de Vigo. Campus de Pontevedra. A Xunqueira, s/n. 36005 PONTEVEDRA. E-mail: xabier.rolan@uvigo.gal 


\begin{abstract}
Instagram allows users to share photos and videos and is a key tool to create destination image. Nowadays destinations compete among them to have a good image and a better position among publics. Therefore, the objectives of this study are to analyze the georeferenced publications of photographs and videos of users in destinations, the reactions they have generated among them and to create an Impact Factor that generates a popularity ranking of the destinations on Instagram. The study shows the contribution of the created Impact Factor in Instagram to measure the popularity of the destinations with respect to previous rankings and their usefulness for the Destination Marketing Organizations.
\end{abstract} tions.

Keywords: Instagram; geotagging; photographs; videos; ranking; popularity; destina-

\title{
1. INTRODUCCIÓN
}

Actualmente los destinos compiten entre sí para tener una buena imagen (Morgan, Pritchard y Pride, 2007) y ser populares, con el objetivo de atraer turistas y riqueza para el territorio. Y para ello necesitan comunicar su marca (Morgan et al., 2007). La marca de los destinos (Blain, Levy y Ritchie, 2005; Crompton, 1992; Morgan, Pritchard y Piggott, 2003) los distingue de la competencia y los hace más atractivos, generando una mejor imagen y una demanda turística superior (Choi et al., 2007; Ekinc y Hosany, 2006; Gallarza et al., 2002). Los destinos con mejor imagen atraen más residentes, mayores inversiones, más estudiantes, más turistas que los quieren ir a visitar y consiguen más eventos como congresos, conciertos, etc. Todo ello genera mayor riqueza y popularidad para el destino (Govers y Go, 2009). Además, está ampliamente reconocido que el turismo supone una importante contribución al desarrollo económico de los territorios (Ordoñez de Pablos yAung, 2017). Por ello, los destinos compiten entre sí cada vez más para estar mejor posicionados que el resto (Drakulic et al., 2017). Un buen posicionamiento aumenta la atractividad de un destino porque lo distingue en la mente de los visitantes potenciales (Ibrahim y Gill, 2005).

Anteriormente los destinos se daban a conocer y se creaban una imagen a través de su presencia en los medios de comunicación, en campañas publicitarias, en ferias de turismo, en libros y en películas o a través de las narraciones y experiencias de conocidos que habían estado en el lugar (Choi, Lehto y Morrison, 2007). Con el surgimiento de Internet los destinos se empezaron a promocionar con un alcance y actualización muy superior a través de sus sitios web (Govers y Go, 2003). Pero con el surgimiento de los medios sociales se ha democratizado la comunicación turística (Jacobsen y Munar, 2012). Ahora no solo las Organizaciones de Marketing de los Destinos (OMDs) comunican. Los usuarios comparten sus experiencias y valoraciones en las plataformas de medios sociales (Jacobsen y Munar, 2012) y los contenidos generados por los usuarios (UGC) tienen una mayor credibilidad (Huertas, Míguez-González y Lozano-Monterru- 
bio, 2017; Huertas y Mariné-Roig, 2018) y un impacto superior en la creación de su imagen que las creadas por las OMDs (Papathanassis y Knolle, 2011).

La mayoría de los medios sociales permite que los usuarios publiquen sus fotografías y vídeos en sus plataformas. Pero, además, los Medios Sociales Visuales (VSM), los especializados en la compartición de fotografías y vídeos, han proliferado ampliamente consiguiendo un gran interés e impacto entre los usuarios (Mull y Lee, 2014). Por ello las fotografías y los vídeos se han convertido en importantes herramientas para la promoción de los destinos turísticos (Hanan y Putit, 2014). Las fotografías pueden mostrar lugares interesantes y atractivos de estos destinos y los vídeos pueden mostrar lo que los usuarios realizan en ellos. Las fotografías que los usuarios cuelgan de los destinos en los medios sociales influyen decisivamente en la imagen que otros usuarios se crean de estos destinos y en su atracción hacia el lugar (Hanan y Putit, 2014; Míguez-González y Huertas, 2015), al igual que ocurre con los vídeos (Huertas, Míguez-González y Lozano-Monterrubio, 2017; Huertas, 2018). Por tanto, crean imagen y popularidad para los destinos.

Instagram es una plataforma que permite publicar fotografías y vídeos y es el VSM que ha experimentado el crecimiento más rápido en los últimos años (Fatanti y Suyadnya, 2015), siendo actualmente uno de los más utilizados por los usuarios. Instagram permite la creación de fotos, vídeos y stories, la edición de imágenes y la publicación instantánea de estas creaciones en los diversos medios sociales (Fatanti y Suyadnya, 2015). Por lo tanto, es una herramienta clave para crear imagen de destino (Huertas, 2018) y para promocionarlos (Hanan y Putit, 2014).

La medición de la influencia digital es una de las cuestiones más interesantes y que más se estudia en la actualidad. Como consecuencia, debido a la influencia que las fotografías y los vídeos de Instagram tienen para generar imagen y popularidad para los destinos (Huertas, 2018), y dado el creciente interés que estos tienen en conseguir un posicionamiento competitivo respecto a los demás (Drakulic et al., 2017; Çakici, Kuhzady y Benli, 2017), ha surgido la necesidad entre los destinos de ser los más fotografiados en Instagram. Por ello se han publicado en Internet rankings de las ciudades del mundo más fotografiadas en Instagram (Diario Las Américas, 2017; TreceBits, 2017) y también de las ciudades españolas (Lázaro, 2017), que según ellos muestran cuáles son las más populares en este medio. Pero estos rankings existentes miden únicamente el número de fotografías tomadas y no tienen en cuenta ni los vídeos, ni las reacciones conseguidas por las publicaciones entre los propios usuarios, ni la desviación que puede suponer la dimensión y el número de habitantes de un territorio en estas cifras.

Por todo ello, este estudio pretende crear una metodología adecuada para medir la popularidad de los destinos en Instagram a través del análisis de las fotografías y los vídeos que comparten los usuarios. A partir de la georreferenciación de las fotografías o vídeos realizados y de un algoritmo creado para la evaluación automatizada, se establecen los territorios más populares en este medio social teniendo en cuenta: el número de habitantes del territorio, el número de fotografías y vídeos publicados y las interacciones que estas y estos generan entre los usuarios. 


\section{MARCO TEÓRICO}

\subsection{Los Medios Sociales Visuales (VSM) en la creación de imagen y popularidad para los destinos}

Los medios sociales han supuesto una importante transformación comunicativa debido a que permiten la generación de contenidos por parte de los usuarios; y ello ha incrementado la información y el conocimiento disponible sobre los destinos turísticos (Hanan y Putit, 2013). Además, sus contenidos generan una gran credibilidad entre los usuarios (Paniagua y Huertas, 2018). Generalmente, las opiniones y valoraciones de otros usuarios sin ningún interés al respecto se consideran mucho más interesantes y creíbles que las informaciones oficiales de los destinos (Mack, Blose y Pan, 2008; Xiang y Gretzel, 2010).

Los medios sociales son importantes creadores de conocimiento (De Bruyn y Lilien, 2008), imagen y popularidad para los destinos (Hanan y Putit, 2013; Cho, Wang y Fesenmaier, 2002; Buhalis y Law, 2008; Lim, Chung y Weaver, 2012; Tham, Croy y Mair, 2013) y, por tanto, también son canales clave para la creación de marcas de destino y para la promoción turística (Fatanti y Suyadnya, 2015). Por ello, actualmente los destinos utilizan estas nuevas tecnologías para conseguir una mejor comunicación de la marca (Jabreel et al., 2018; Lalicic et al., 2018), una mejor imagen (Morgan, Pritchard y Pride, 2007) y atracción de turistas (Pike y Page, 2014).

Los medios sociales basados en la publicación de fotografías y vídeos (VSM), y entre todos ellos principalmente Instagram, han proliferado mucho recientemente y tienen un gran éxito entre los usuarios (Mull y Lee, 2014). Desde finales de 2016 Instagram consigue 600 millones de usuarios nuevos cada mes y se estima que se comparten más de 80 millones de fotos al día en este medio (Hendrickse et al., 2017; Çakici, Kuhzady y Benli, 2017).

En España y en el Mundo, Instagram es uno de los medios sociales con mayor crecimiento y popularidad entre los usuarios, especialmente entre los segmentos más jóvenes. Instagram permite a los usuarios la creación de fotos, vídeos y stories, la edición de imágenes, la compartición instantánea de estas creaciones en su plataforma y en los diversos medios sociales y las interacciones de otros usuarios (Fatanti y Suyadnya, 2015; Huertas, 2018). Instagram posee un gran potencial para la visualización y permite la georreferenciación, o sea, la ubicación y geolocalización de los contenidos que se comparten, lo cual fomenta la promoción de los lugares, porque con la georreferenciación los individuos pueden encontrar fácilmente fotografías o vídeos de los lugares que deseen (Fatanti y Suyadnya, 2015).

En el ámbito turístico, Instagram es una herramienta clave para promocionar los destinos (Hanan y Putit, 2013). Por ello muchos de ellos se promocionan utilizando Instagram proactivamente. Pero Instagram además también permite que los usuarios, a través de sus fotografías, vídeos y stories personales promocionen los destinos de todo el mundo (Fatanti y Suyadnya, 2015).

Los Medios Sociales Visuales son herramientas especialmente potentes para promocionar los destinos y aumentar su popularidad (Hanan y Putit, 2013), ya que las fotografías muestran lugares de interés, actividades en el lugar e incitan a visitarlo. A mayor número de fotografías, aumenta el conocimiento y la imagen del destino entre los usuarios y, por 
tanto, también su atractividad (Fatanti y Suyadnya, 2015). Existe una corriente de estudios centrada en analizar el impacto de los medios sociales en la imagen que se crean los usuarios de los destinos (Seabra, Abrantes y Lages, 2007, Lim, Chung y Weaver, 2012; Llodrà-Riera et al., 2015) y algunos estudios se centran ya exclusivamente en el rol de Instagram (Fatanti y Suyadnya, 2015; Molinillo et al., 2018).

Los usuarios se crean las imágenes de los destinos influidos por los contenidos de otros usuarios (UGC) que comparten experiencias e información en los medios sociales (LlodràRiera et al., 2015). Así co-crean la imagen de los destinos (Aitken y Campelo, 2011; Kavaratzis, 2012; Ho, Lin y Chen, 2012) e influyen en las futuras decisiones turísticas de otros. Pero Instagram, debido a sus características, a la edición que permite de las imágenes y al potencial de participación que ofrece, es el medio social que establece una mejor relación con los usuarios. Además, al contener principalmente imágenes requiere menor esfuerzo de los usuarios para procesarlas y comporta la creación de una mejor imagen para el destino, así como la generación de mayores intenciones de visita (Molinillo et al., 2018).

Las imágenes creadas sobre un lugar en las mentes de los turistas potenciales influyen decisivamente en la elección del destino (Kim, Kim y Bolls, 2014). Según Lee y Gretzel (2012), los turistas que se han creado una imagen de un destino tienen muchas más probabilidades de que consideren visitarlo. De igual modo, los destinos mejor posicionados en la mente de los individuos son los que tienen probabilidades más altas de ser seleccionados para ser visitados (Chen y Lin, 2012; Pike, 2017). Según Pike (2009), el 70\% de los turistas internacionales viajan solo a diez países y el resto de países compiten entre sí para repartirse el $30 \%$ de turistas restante. Por ello, es clave posicionarse entre las primeras posiciones de popularidad de los destinos (Qu, Kim e Im, 2011) y por ello también son necesarios los rankings de popularidad.

Pero obtener un buen posicionamiento y una imagen distintiva en la mente de los turistas potenciales (Gallarza, Saura y García, 2002; Pike, 2012), como afirman Kuzhzady, Ghasemi y Hashemi (2017), es un proceso complejo (Fyall y Garrod, 2005; Shwaighofer, 2013) en el cual se deben tangibilizar aspectos intangibles de los destinos. Por ello son tan importantes las fotografías y los vídeos en este proceso, porque visualizan e incrementan la tangibilidad (Tasci, 2008; Tasci y Gartner, 2007). El 47\% de los usuarios han visitado destinos basando su elección en las fotografías publicadas por amigos y conocidos (Khlat, 2014).

Çakici, Kuhzady y Benli (2017), haciendo un análisis de las fotografías turísticas de los destinos realizadas por usuarios, demostraron que un mayor número de fotografías tomadas por los usuarios en ciertas atracciones de un destino significaba una mayor popularidad de estas atracciones entre los turistas. El simple hecho de aumentar la presencia en las fotografías ya aumentaba la presencia en la mente de los individuos y aumentaba la imagen del lugar. Lo mismo ocurría con el número de me gusta y comparticiones, que significaban un mayor engagement por parte de los usuarios y un mayor agrado del lugar entre ellos.

Cabe destacar que las fotografías y vídeos creados por todo tipo de usuarios (turistas, residentes, u otros) pueden ser de contenido positivo o negativo (Trusov, Bucklin y Pauwels, 2009), y por tanto, también podrían tener una influencia negativa en la cocreación de la imagen de los destinos. Con todo, estudios anteriores (Huertas, 2018) han demostrado que los usuarios no utilizan Instagram para mostrar fotografías ni vídeos negativos cuando van de viaje, sino que muestran mayoritariamente aspectos y situacio- 
nes positivas en los destinos. Así pues, Instagram maximiza la imagen de los destinos (Huertas, 2018) y la popularidad de los territorios. Además, un estudio de Vermeulen y Seegers (2009) que analizaba los comentarios negativos de los usuarios en alojamientos demostró que, a pesar de la negatividad de los comentarios, estos también aumentaban el conocimiento de los alojamientos entre los usuarios y, por tanto, los tenían más presentes para futuras decisiones turísticas. Así pues, demostraron que los comentarios negativos tampoco tenían un impacto tan negativo en la imagen y las decisiones turísticas, sino que aumentaban la presencia y el posicionamiento de los alojamientos en la mente de los usuarios.

\subsection{El rol de las fotografías y los vídeos digitales de los usuarios en la creación de la imagen de los destinos}

Las fotografías son un elemento clave en la comunicación de los destinos (Morgan y Pritchard, 1998; Míguez-González y Huertas, 2015), porque acercan el territorio a los usuarios virtuales y poseen una gran fuerza en la comunicación de valores y la creación de experiencias. Diversos estudios desde el ámbito de la comunicación han demostrado que las fotografías poseen un gran potencial para generar percepciones y creación de imagen (Coombs y Holladay, 2011; Zillman, Gibson y Sargent, 1999). Diversos estudios han mostrado el rol determinante de las fotografías en la configuración de la imagen de los destinos (Jenkins, 2003; un sMarkwell, 1997; Míguez-González y Huertas, 2015).

Por ello, las fotografías turísticas sirven a los investigadores para analizar y conocer tanto qué les atrae más, como las percepciones y actitudes de los turistas hacia los destinos (Balomenou, Garrod y Georgiadou, 2017). La mayoría de los estudios tratan de analizar, con muchas limitaciones, el contenido de las fotografías ya sea con métodos cuantitativos, pero utilizando codificaciones manuales (Pearce, Wu y Chen, 2015); o con sistemas más automatizados, como el Análisis de Componentes Principales (ACP) o el Análisis de Correlación Canónica (ACC) (Balomenou, Garrod y Georgiadou, 2017).

Los vídeos también son importantes herramientas para la comunicación de la identidad de los destinos y ayudan a crear su imagen (Lim, Chung y Weaver, 2012; Kim, Kim y Bolls, 2014; Huertas, Míguez-Gonzáles y Lozano-Monterrubio, 2017). Su potencial se basa en que la información visual está directamente conectada con el proceso interno de la percepción (Hsieh y Chen, 2011; Kim, Kim y Bolls, 2014). Por tanto, los vídeos generan experiencias emocionales más potentes, ideales para la comunicación de los destinos turísticos. Además, el vídeo reproduce a través de una continuidad visual la experiencia del turista y muestra las actividades que se pueden hacer en el lugar (Dinhopl y Gretzel, 2015). Los estudios académicos sobre vídeos en el ámbito turístico han analizado su impacto en la experiencia turística (Jansson, 2002; Tussyadiah y Fesenmaier, 2009; Dinhopl y Gretzel, 2015), en la creación de la imagen de los destinos (Trusov, Bucklin y Pauwels, 2009; Lim, Chung y Weaver, 2012) o en las decisiones turísticas que acaban tomando otros usuarios (Kim, Kim y Bolls, 2014).

El avance de la tecnología ha facilitado la expansión de las fotografías y los vídeos de los usuarios que, gracias a las nuevas aplicaciones de las plataformas, han proliferado en los medios sociales (Dinhopl y Gretzel, 2015). Por ello recientemente, a pesar de que 
existen estudios que analizan los vídeos y las fotografías que comparten las OMDs en sus plataformas, que son fuentes "inducidas" (Balomenou, Garrod y Georgiadou, 2017; Çakici, Kuhzady y Benli (2017), han aumentado los estudios que analizan las fotografías y los vídeos creados y compartidos por los propios usuarios, que provienen de fuentes “orgánicas" (Tussyadiah y Fesenmaier, 2009; Trusov, Bucklin y Pauwels, 2009; Dinhopl y Gretzel, 2015; Huertas, 2018). Actualmente estas fuentes "orgánicas" son las más numerosas y tienen un gran poder de influencia en otros usuarios (Kuhzady, Ghasemi y Hashemi, 2017), convirtiéndose en co-creadoras de la imagen de los destinos.

Los estudios que analizan las fotografías de los usuarios en los destinos a través de la georreferenciación o geolocalización (Williams, 2015) permiten mostrar en un mapa los lugares más fotografiados del territorio. La imagen de los destinos no se debería entender a través de los modelos tradicionales de representación, sino a través de las fotografías que los usuarios hacen y comparten en el propio lugar (Cosgrove, 2005), ya que los turistas muestran puntos de interés distintos a los mostrados por las OMDs (Stepchenkova y Zhan, 2013). A través de las fotografías georreferenciadas, los usuarios co-crean una imagen colectiva de los destinos (Cosgrove, 2005).

Además, la implicación, la participación y las reacciones de los usuarios a las fotografías y vídeos de otros que se comparten en los medios sociales también son importantes en la creación de la imagen de los destinos (Molinillo et al., 2018). Estudios anteriores han demostrado que la implicación de los usuarios con los contenidos generados en los medios sociales tiene un impacto positivo en la creación de la imagen de los destinos (Lu, Chi y Liu, 2015; Prayag y Ryan, 2012). De hecho, una mayor implicación genera un mayor impacto en la creación de una imagen positiva de destino (Martín-Santamaría, Beerli-Palacio y Nazzareno, 2017). Se ha demostrado que el contenido visual de por sí también genera mayor engagement entre los usuarios (Mariani, Di Felice y Mura, 2016; Huertas, Setó-Pàmies y Míguez-González, 2015). Además, los comentarios y la interactividad de los usuarios con las fotografías y vídeos generan una mayor implicación (Tussyadiah y Fesenmaier, 2009), que a su vez aumenta la tangibilidad y la decisión de visita (Fasolo et al., 2006; Koernig, 2003). Por tanto, la implicación, el engagement y la interactividad de los usuarios respecto a las fotografías y vídeos de otros usuarios son importantes y se deben tener en cuenta para la medición de la imagen y la popularidad de los destinos.

\section{METODOLOGÍA}

Así pues, a partir de la georreferenciación los objetivos de esta investigación son:1analizar las fotografías y vídeos de los usuarios de ciertos destinos etiquetadas en Instagram para conocer el volumen de las publicaciones, cuándo se publican más y por qué y qué interacciones generan entre los usuarios; y 2-teniendo en cuenta estas variables previas de análisis y el número de habitantes de los territorios, crear a través de un algoritmo un factor de impacto que nos permita construir un ranking para valorar qué destinos consiguen mayor popularidad e imagen en Instagram a través de las fotografías y vídeos generados por los usuarios. 


\subsection{Selección de la muestra}

La muestra se acotó a las ciudades españolas que superan el medio millón de habitantes, que son: Barcelona, Madrid, Málaga, Sevilla, Valencia y Zaragoza. Se trataba de seleccionar destinos que generaran un elevado volumen de publicaciones de fotografías y vídeos georreferenciados de los usuarios, suficiente como para elaborar un ranking con un elevado volumen de datos y gran fiabilidad.

Pero precisamente debido a la cantidad de fotografías y vídeos obtenidos, el periodo de investigación se acotó a dos semanas naturales en dos épocas del año muy distintas: una semana del 11 al 17 de diciembre de 2017, ambos inclusive, y otra semana del 4 al 10 de junio de 2018, ambos también inclusive. De este modo se podía estudiar la tendencia semanal en invierno y en verano sin injerencias puntuales derivadas de festividades nacionales o locales.

Se recogieron todas las fotografías y vídeos de usuarios georreferenciados existentes de cada destino. La muestra total de fotografías y vídeos analizados para las dos semanas del estudio se puede ver en la tabla siguiente:

Tabla 1

\section{MUESTRA DE LA INVESTIGACIÓN}

\begin{tabular}{|c|c|c|c|c|}
\hline Destinos & $N^{o}$ habitantes & $\begin{array}{l}N^{o} \text { Publicaciones } \\
\text { (foto y vídeo) }\end{array}$ & $\begin{array}{l}N^{o} \text { de fotografías } \\
\text { analizadas }\end{array}$ & $\begin{array}{l}N^{o} \text { de vídeos } \\
\text { analizados }\end{array}$ \\
\hline Madrid & 3.165 .541 & 119.508 & 111.362 & 8.146 \\
\hline Barcelona & 1.608 .746 & 145.457 & 145.296 & 9.161 \\
\hline Valencia & 790.201 & 29.904 & 28.186 & 1.718 \\
\hline Sevilla & 690.566 & 31.544 & 29.869 & 1.674 \\
\hline Zaragoza & 661.108 & 23.635 & 22.198 & 1.437 \\
\hline Málaga & 569.009 & 10.602 & 9.980 & 622 \\
\hline
\end{tabular}

Fuente: Elaboración propia a partir de los datos del INE2016 y la muestra resultante de la recogida de datos.

Como se observa, los destinos de la muestra son muy dispares en cuanto a volumen de población. Mientras los cuatro con menor número de habitantes (Valencia, Sevilla, Zaragoza y Málaga) no muestran grandes diferencias poblacionales entre ellos, Barcelona dobla el número de habitantes de Valencia, que ocupa la tercera posición, y Madrid prácticamente dobla el de Barcelona ocupando la primera posición en volumen poblacional.

Por lógica, es de suponer que a mayor número de habitantes los destinos obtendrán mayor número de fotografías y vídeos, ya que al haber más individuos en el lugar, existe una mayor probabilidad de que se realicen más fotografías y vídeos. Pero si observamos el número de publicaciones recogidas en las dos semanas del estudio, cabe destacar que a pesar de que Madrid prácticamente duplica la población de Barcelona, esta supera a 
Madrid tanto en el número de fotografías como en el número de vídeos. También cabe destacar que a pesar de que Valencia posee unos 100.000 habitantes más que Sevilla, este último destino consigue 1.600 fotografías más que Valencia.

Como se observa, en general el número de vídeos es muy inferior al de fotografías para todos los destinos. Este hecho tiene una explicación lógica, ya que las fotografías son muy fáciles y rápidas de realizar, mientras que la confección y visualización de los vídeos son más costosas. La diferencia en el número de vídeos entre Madrid y Barcelona es muy inferior al de fotografías, ya que Barcelona tan solo supera a Madrid en 1.000 vídeos. Con todo, se debe tener en cuenta que Madrid dobla la población de Barcelona. El número de vídeos de Valencia en este caso sí supera al número de Sevilla, como cabría esperar por el superior número de habitantes, aunque por muy poca diferencia. Y finalmente, cabe destacar que Málaga obtiene un número de vídeos muy inferior al resto de las ciudades de la muestra.

El proceso de captura de los datos (las fotografías y vídeos de los usuarios georreferenciadas de cada destino) se llevó a cabo con la herramienta Instabro: una aplicación para OSX que se conecta a la API de Instagram y realiza consultas de hasta 500 items, pero de forma acumulativa. Instabro permite exportar los metadatos de las fotografías geoetiquetadas en cualquiera de las ubicaciones registradas en la red social de fotografía. La segmentación basada en geoetiquetas es muy pertinente para este estudio, dado que se trata de un metadato que solo aparece cuando la fotografía tomada contiene información de la ubicación. Así Instagram puede ofrecer lugares etiquetables cercanos.

\subsection{Método de análisis}

Las variables analizadas en este estudio son: 1- el volumen de publicaciones según el tipo de contenido publicado (si son fotografías o vídeos); 2- las reacciones o interacciones que estas publicaciones de los usuarios reciben de otros usuarios especificando si son visualizaciones, me gusta y comentarios; 3- la fecha de publicación de estos contenidos y la distribución temporal de estas publicaciones; y 4- el Factor de Impacto en Instagram para la creación del ranking de popularidad de los territorios o los destinos.

Para el tratamiento de datos y su análisis se empleó una hoja de cálculo estadístico. Se analizaron frecuencias, porcentajes y el promedio/día para el cálculo de las interacciones o reacciones de los usuarios, porque se trata de una métrica muy adecuada para analizar el promedio de reacciones teniendo en cuenta el número de publicaciones existentes.

El Factor de Impacto en Instagram (en adelante, FII) es una herramienta metodológica elaborada para este estudio que parte de la creación de un algoritmo y permite la obtenciónde un ranking de los destinos más populares en Instagram a partir de las fotografías y vídeos publicados por los usuarios. El algoritmo utiliza y relaciona las variables del estudio. Concretamente relaciona el promedio diario de las publicaciones georreferenciadas y las interacciones recibidas por parte de los usuarios (reproducciones, me gusta y comentarios) con el número de habitantes de la ciudad en cuestión.

El algoritmo creado se expresa en la siguiente fórmula:

$$
F I I=\text { Promedio diario }(P+R+L+C) \div P o b
$$


Donde $\mathrm{P}=$ el número de publicaciones de fotografías y vídeos de los usuarios para un determinado destino, $\mathrm{R}=$ el número de reproducciones o visualizaciones solo de los vídeos publicados, $\mathrm{L}=$ el número de me gusta conseguidos por el conjunto de publicaciones (fotografías y vídeos), $\mathrm{C}=$ el número de comentarios obtenidos por el conjunto de publicaciones (fotografías y vídeos) y Pob= el número de habitantes o volumen poblacional del destino de acuerdo con los datos del Instituto Nacional de Estadística.

Como se observa, el FII no es una simple contabilización del número de fotografías y vídeos publicados por los usuarios, sino que es una herramienta más compleja y mucho más adecuada para establecer un ranking de popularidad de destinos en Instagram, ya que también tiene en cuenta las reproducciones o visualizaciones, las reacciones de los usuarios y la población del territorio. Así pues, el valor del FII indica mayor popularidad o mejor posicionamiento cuanto más elevado es el resultado, lo cual muestra que el destino está mejor posicionado o es más popular entre los usuarios.

El empleo de la variable poblacional otorga proporcionalidad y coherencia a los resultados en tanto es más probable que un mayor volumen de actividad se produzca en destinos con un mayor volumen de población. En este sentido, se ha optado por emplear los datos censales. No se ha estimado la población flotante por la dificultad de obtener estos datos de los destinos durante las dos semanas concretas de análisis.

\section{RESULTADOS}

El uso georreferenciado de Instagram en la muestra analizada de los destinos españoles revela interesantes cuestiones desde el punto de vista cuantitativo. En la tabla 2 se muestran las frecuencias o el total de fotografías y vídeos georreferenciados en estos destinos durante las dos semanas seleccionadas para la investigación. Los porcentajes se han analizado sobre el total de fotografías y vídeos por el mismo periodo y destino. Así, se puede observar si los destinos poseen mayores porcentajes de fotografías o de vídeos.

\section{Tabla 2 \\ FRECUENCIAS DE FOTOGRAFÍAS Y VÍDEOS Y PORCENTAJES RESPECTO AL TOTAL DE PUBLICACIONES}

Destinos Frec.Fotos 2017 Frec.Fotos 2018 Frec. Vídeos 2017 Frec. Vídeos 2018 (y\% sobre el total) $\quad$ (y\% sobre el total) $\quad$ (y\% sobre el total) $\quad(y \%$ sobre el total)

\begin{tabular}{r|rrrr}
\hline Barcelona & $49469(93.73 \%)$ & $86827(93.69 \%)$ & $3309(6.27 \%)$ & $5852(6.31 \%)$ \\
Madrid & $43417(93.35 \%)$ & $67945(93.08 \%)$ & $3093(6.65 \%)$ & $5053(6.92 \%)$ \\
Valencia & $9222(94.31 \%)$ & $18964(94.23 \%)$ & $556(5.69 \%)$ & $1162(5.77 \%)$ \\
Sevilla & $11684(94.77 \%)$ & $18185(94.64 \%)$ & $644(5.22 \%)$ & $1030(5.36 \%)$ \\
Málaga & $8059(93.68 \%)$ & $14138(94.05 \%)$ & $544(6.32 \%)$ & $894(5.95 \%)$ \\
Zaragoza & $3803(94.37 \%)$ & $6176(93.98 \%)$ & $226(5.62 \%)$ & $396(6.02 \%)$
\end{tabular}

Fuente: Elaboración propia. 
Si observamos en primer lugar las frecuencias de las fotografías en el periodo invernal (diciembre 2017) y las comparamos con las del período estival (junio 2018), como es de esperar, las frecuencias de todos los destinos analizados aumentan y son superiores en junio que en diciembre. Ello se explica lógicamente por la existencia en junio de un mejor clima y más horas de sol, que influyen en que todo tipo de públicos (sean turistas o incluso residentes) estén más tiempo fuera de casa, hagan más actividades y por ello también se obtengan más fotografías. Con todo, es innegable el impacto del turismo en estos datos. Debido a que en junio hay muchos más turistas en los destinos analizados, estos con sus fotografías turísticas elevan el número obtenido. En algunos destinos, como Barcelona, Valencia, Zaragoza o Málaga, las frecuencias de fotografías en la semana de junio casi doblan las de diciembre. Pero las diferencias entre los dos períodos en Madrid y Sevilla no son tan amplias. Ello puede significar que estos dos destinos poseen mucho turismo durante todo el año acusando menos la estacionalidad, ya que ninguno de los dos son destinos de costa y, por tanto, no se ven afectados por el turismo de sol y playa de la época estival.

Frecuencias similares se observan para los vídeos de los dos períodos analizados, aunque con menores diferencias entre Madrid y Barcelona y el resto de destinos, debido a que el número de vídeos es muy inferior al de fotografías para todos los destinos analizados. Cabe destacar que para la semana de diciembre de 2017 el número de vídeos es superior en Sevilla que en Valencia a pesar de tener menos habitantes. Ello coincide con el argumento anterior de que Sevilla es un destino que posee turismo durante todo el año y no se ve tan influido por el turismo estacional de sol y playa.

Si nos centramos ahora en los porcentajes respecto al total de publicaciones conseguidas, observaremos las diferencias entre porcentajes de fotografías y de vídeos por cada destino y periodo. En general, los porcentajes son muy uniformes para la toda la muestra del análisis y apenas hay diferencias significativas. Por tanto, se puede afirmar para todos los destinos que el 93-94\% de los contenidos que se suben a Instagram son fotografías frente al 5-6\% de vídeos. Sobre este porcentaje se mueven Madrid, el destino que tiene un porcentaje superior de vídeos (hasta un 6’92\% de las publicaciones) y Sevilla, el destino con un porcentaje superior de fotografías (con un 94'77\%).

Si comparamos los porcentajes de los destinos por periodo, todos ellos excepto Málaga aumentan ligeramente los porcentajes de vídeos la semana de junio respecto a la de diciembre, mientras que disminuyen también muy ligeramente los porcentajes de las fotografías. Ello puede tener relación con el mayor número de actividades al aire libre, que seguro serán superiores en junio debido a las condiciones climatológicas.

El destino con superior porcentaje de fotografías es Sevilla, especialmente en diciembre de 2017 con un porcentaje de $94.77 \%$ y en junio con $94.64 \%$. Le sigue Zaragoza con un $94.37 \%$ en diciembre y Valencia con un $94.31 \%$ en diciembre y $94.23 \%$ en junio. Ellos poseen un índice ligeramente superior de fotografías que de vídeos. En cambio, Madrid es el destinocon un porcentaje superior de vídeos (6.92\% en junio de 2018 y $6.65 \%$ en diciembre de 2017), seguido por Málaga en diciembre (con un 6.32\%) y Barcelona en las dos épocas (6.31\% en junio 2018 y $6.27 \%$ en diciembre 2017). Ello no significa que consiguen más vídeos que fotografías, sino que en proporción poseen un 
porcentaje un poco superior de vídeos respecto al de fotografías que el resto de los destinos. Ello quizás se explique por una oferta superior de ocio y actividades en el territorio.

Unas variables importantes que miden el éxito de las fotografías y los vídeos en Instagram y en todos los medios sociales son las reacciones que estas y estos consiguen por parte de otros usuarios, ya que indican interés por su parte (con las reproducciones), agrado (con los me gusta) o ganas de participar u opinar (con los comentarios). Por tanto, en la Tabla 3 se muestran: las frecuencias totales del conjunto de fotos y vídeos publicados, la frecuencia total de las reproducciones conseguidas por los vídeos publicados (ya que es una medida que sólo se puede obtener de los vídeos), la frecuencia total de me gusta y de comentarios conseguidos para todas las fotografías y vídeos publicados; así como el promedio por día y publicación de cada una de estas frecuencias entre paréntesis.

\section{Tabla 3}

\section{REACCIONES DE LOS USUARIOS}

Frecuencias de publicaciones conjuntas de fotos $\mathrm{y}$ vídeos, frecuencias de reproducciones solo de los vídeos, frecuencias de me gusta de fotos y vídeos y comentarios de fotos y vídeos (y los promedios por día y publicaciones) de los destinos en los dos períodos de captura de datos $(2017$ y 2018)

\begin{tabular}{|c|c|c|c|c|c|c|c|}
\hline & & Madrid & Barcelona & Valencia & Sevilla & Málaga & Zaragoza \\
\hline \multirow{4}{*}{2017} & $\begin{array}{l}\text { Publicaciones - foto y } \\
\text { vídeo (promedio día) }\end{array}$ & $\begin{array}{l}46.510 \\
(6.644)\end{array}$ & $\begin{array}{l}52.778 \\
(7.540)\end{array}$ & $\begin{array}{c}9.778 \\
(1.397)\end{array}$ & $\begin{array}{l}12.329 \\
(1.761)\end{array}$ & $\begin{array}{c}8.603 \\
(1.229)\end{array}$ & $\begin{array}{l}4.030 \\
(576)\end{array}$ \\
\hline & $\begin{array}{l}\text { Reproducciones vídeos } \\
\text { (promedio día) }\end{array}$ & $\begin{array}{l}2.329 .991 \\
(753)\end{array}$ & $\begin{array}{l}2.676 .409 \\
(809)\end{array}$ & $\begin{array}{c}560.728 \\
(1009)\end{array}$ & $\begin{array}{l}536.863 \\
(834)\end{array}$ & $\begin{array}{l}300.015 \\
\quad(551)\end{array}$ & $\begin{array}{l}173.633 \\
(768)\end{array}$ \\
\hline & $\begin{array}{l}\text { Me gusta, fotos y } \\
\text { vídeos (promedio día) }\end{array}$ & $\begin{array}{l}6.318 .618 \\
(136)\end{array}$ & $\begin{array}{l}7.413 .300 \\
(140)\end{array}$ & $\begin{array}{l}1.632 .015 \\
(167)\end{array}$ & $\begin{array}{l}1.610 .913 \\
(131)\end{array}$ & $\begin{array}{l}1.298 .824 \\
\quad(151)\end{array}$ & $\begin{array}{l}555.502 \\
(138)\end{array}$ \\
\hline & $\begin{array}{l}\text { Comentarios fotos y } \\
\text { vídeos (promedio día) }\end{array}$ & $\begin{array}{l}184.874 \\
(4)\end{array}$ & $\begin{array}{l}195.541 \\
(4)\end{array}$ & $\begin{array}{l}184.874 \\
(19)\end{array}$ & $\begin{array}{c}37.488 \\
(3)\end{array}$ & $\begin{array}{c}36.854 \\
(4)\end{array}$ & $\begin{array}{l}29.256 \\
(7)\end{array}$ \\
\hline \multirow{4}{*}{2018} & $\begin{array}{l}\text { Publicaciones - foto y } \\
\text { vídeo (promedio día) }\end{array}$ & $\begin{array}{c}72.998 \\
(10.428)\end{array}$ & $\begin{array}{c}92.679 \\
(13.240)\end{array}$ & $\begin{array}{l}20.126 \\
(2.875)\end{array}$ & $\begin{array}{l}19.215 \\
(2.745)\end{array}$ & $\begin{array}{l}15.032 \\
(2.147)\end{array}$ & $\begin{array}{l}6.572 \\
(939)\end{array}$ \\
\hline & $\begin{array}{l}\text { Reproducciones vídeos } \\
\text { (promedio día) }\end{array}$ & $\begin{array}{l}19.315 .984 \\
(3823)\end{array}$ & $\begin{array}{l}9.207 .377 \\
(1573)\end{array}$ & $\begin{array}{l}1.203 .880 \\
(1036)\end{array}$ & $\begin{array}{l}974.367 \\
(946)\end{array}$ & $\begin{array}{l}659.575 \\
(738)\end{array}$ & $\begin{array}{c}112.541 \\
(284)\end{array}$ \\
\hline & $\begin{array}{l}\text { Me gusta, fotos y } \\
\text { vídeos (promedio día) }\end{array}$ & $\begin{array}{l}15.278 .802 \\
(209)\end{array}$ & $\begin{array}{c}17.521 .355 \\
(189)\end{array}$ & $\begin{array}{l}3.197 .497 \\
\quad(159)\end{array}$ & $\begin{array}{l}2.442 .989 \\
\quad(127)\end{array}$ & $\begin{array}{l}2.017 .350 \\
\quad(134)\end{array}$ & $\begin{array}{c}808.893 \\
(123)\end{array}$ \\
\hline & $\begin{array}{l}\text { Comentarios fotos y } \\
\text { vídeos (promedio día) }\end{array}$ & $\begin{array}{l}462.018 \\
(6)\end{array}$ & $\begin{array}{l}454.220 \\
\quad(5)\end{array}$ & $\begin{array}{l}104.707 \\
(5)\end{array}$ & $\begin{array}{l}82.730 \\
(4)\end{array}$ & $\begin{array}{l}68.763 \\
(5)\end{array}$ & $\begin{array}{l}36.455 \\
(6)\end{array}$ \\
\hline
\end{tabular}

Fuente: Elaboración propia.

Como se observa en la tabla 3, las reproducciones o visualizaciones de los vídeos (segunda columna para cada periodo) tienen unas frecuencias y unos promedios/día/ vídeo elevados para los dos destinos con mayor población y con mayor número de publicación 
de vídeos, que son Madrid y Barcelona. Para el 2018 las reproducciones de los vídeos de estas dos ciudades aumentan muchísimo respecto al 2017, especialmente las de los vídeos de Madrid (hasta ocho veces más), que superan la duplicación de las reproducciones de Barcelona. Así pues, aunque Barcelona consiguió más vídeos publicados y georreferenciados en Instagram que Madrid, en junio de 2018 Madrid consiguió muchas más reproducciones o visualizaciones, lo cual es un dato importante para la difusión de estos vídeos que indica el éxito de su publicación.

Con todo, los índices que en mayor medida indican el éxito de las publicaciones entre los usuarios, mostrando su agrado, su implicación y un impacto superior en la imagen que se crean de los destinos (Molinillo et al., 2018; Lu, Chi y Liu, 2015; Prayag y Ryan, 2012) son los me gusta y los comentarios que consiguen de otros usuarios. Los me gusta muestran claramente el agrado de los usuarios respecto a las publicaciones. En la tabla 3 se observa que en este indicador Barcelona consigue más me gusta que Madrid en los dos períodos. Así pues, sus publicaciones y lo que muestran gustan más y son más populares entre los usuarios. En el 2018 Madrid, Barcelona y Valencia, que son los destinos que obtienen más me gusta con sus publicaciones prácticamente doblan el número de sus frecuencias y promedios/día/publicación de me gusta obtenidos respecto al 2017.

Cabe destacar que los promedios/día/publicación de me gusta, que son los datos que se muestran entre paréntesis, son bastante elevados para los destinos que no obtienen tantas publicaciones, porque son indicadores que miden el promedio de me gusta conseguidos por las publicaciones realizadas. Así pues, se muestra que hay destinos como Valencia en 2017, que a pesar de no tener tantas publicaciones como Barcelona o Madrid, consiguen un promedio superior de me gusta por publicación. Ello es un indicador importante del éxito de las publicaciones (Tussyadiah y Fesenmaier, 2009) aunque estas sean inferiores en número.

Los comentarios son muy inferiores a los me gusta y se observa que, excepto los de Valencia, todos aumentan en la semana de junio de 2018 respecto a diciembre de 2017. Cabe destacar que en el periodo analizado del 2018 los comentarios en Madrid y Barcelona se duplican, siendo incluso superiores en Madrid que en Barcelona para ese año. En cambio, si observamos el promedio de comentario por día y por publicaciones conseguidas, destaca Valencia, que en el 2017 consiguió un promedio elevado respecto al número total de publicaciones que posee; y Zaragoza que, a pesar de tener menos publicaciones, consigue un número considerable de comentarios y por tanto, el promedio publicación y día es elevado.

La figura 1 recoge el volumen de publicaciones e interacciones para cada ciudad y periodo analizado mostrando su evolución para cada día de la semana.

Si observamos la figura 1, lo primero que se aprecia es que las publicaciones (en la parte inferior del gráfico) por lo general son bastante homogéneas al largo de la semana, aumentando todas ligeramente el fin de semana especialmente para el periodo de 2017 . Ello se debe a que en invierno es durante el fin de semana cuando hay más ocio, tiempo libre y aumenta el turismo. En cambio, para el periodo de junio de 2018 el incremento del fin de semana es menor. 


\section{Figura 1 \\ EVOLUCIÓN SEMANAL DEL VOLUMEN DE PUBLICACIONES \\ E INTERACCIONES \\ 2017 (línea continua) y 2018 (línea discontinua)}

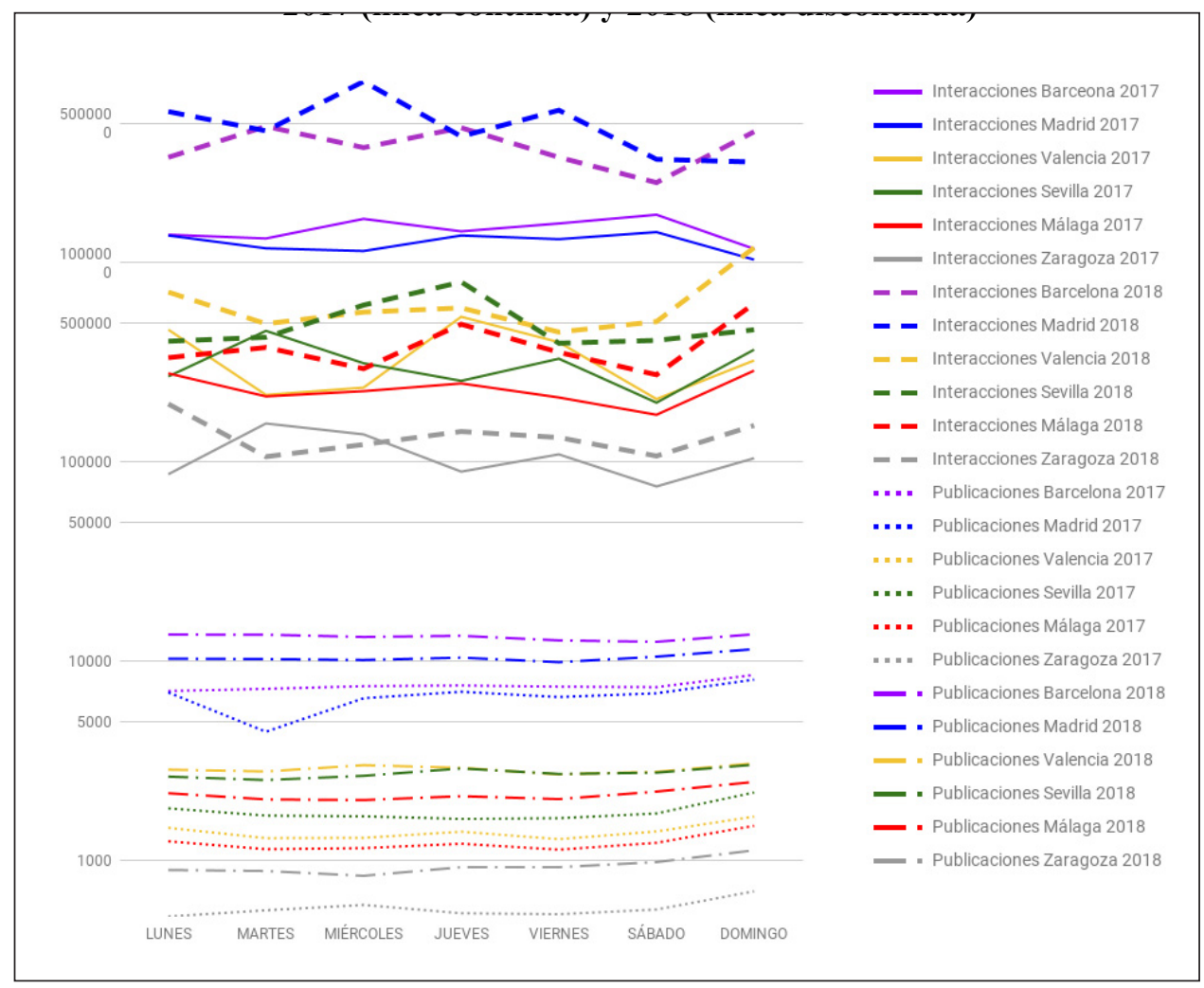

Fuente: Elaboración propia.

Como se observa, las publicaciones son bastante homogéneas excepto el descenso de publicaciones en Madrid el martes de la semana de diciembre de 2017. Pero lo que resulta mucho más variable son las reacciones de los usuarios (en la parte superior del gráfico), que muestran el impacto que realmente obtuvieron las publicaciones. Como aspecto a destacar podemos decir que mientras las publicaciones suben el fin de semana, las reacciones de los usuarios a dichas publicaciones aumentan en mitad de semana y disminuyen mayoritariamente en sábado, para recuperarse el domingo. Madrid tiene picos de reacciones elevados para el 2018 en miércoles y viernes, mientras que Barcelona, Sevilla, Málaga y Valencia aumentan el jueves. Así pues, se observan tendencias distintas entre las publicaciones y las reacciones a las publicaciones por parte de los usuarios.

Finalmente, si analizamos el ranking creado a partir del Factor de Impacto en Instagram FII (figura 2), que además de las publicaciones tiene en cuenta el volumen poblacional y 
las reacciones de los usuarios, se observa que Barcelona ocupa la primera posición con gran diferencia respecto a Madrid. Ello es debido a que Barcelona obtiene más publicaciones tanto de fotografías como de vídeos que Madrid teniendo prácticamente la mitad de población. Además, a ello se añade el mayor número de reacciones de los usuarios a estas publicaciones, especialmente con el número de me gusta conseguidos. Así pues, mientras el número de publicaciones entre Madrid y Barcelona no son tan distantes (véase figura 3 para poder hacer la comparativa), el FII y el ranking muestran la popularidad o el mejor posicionamiento de Barcelona con mayor diferencia respecto a Madrid, pero con menor distancia respecto al resto de ciudades debido también a que el FII tiene en cuenta el volumen poblacional.

\section{Figura 2}

\section{VALORES DEL FII Y RANKING DE DESTINOS CON MAYOR IMPACTO EN INSTAGRAM}

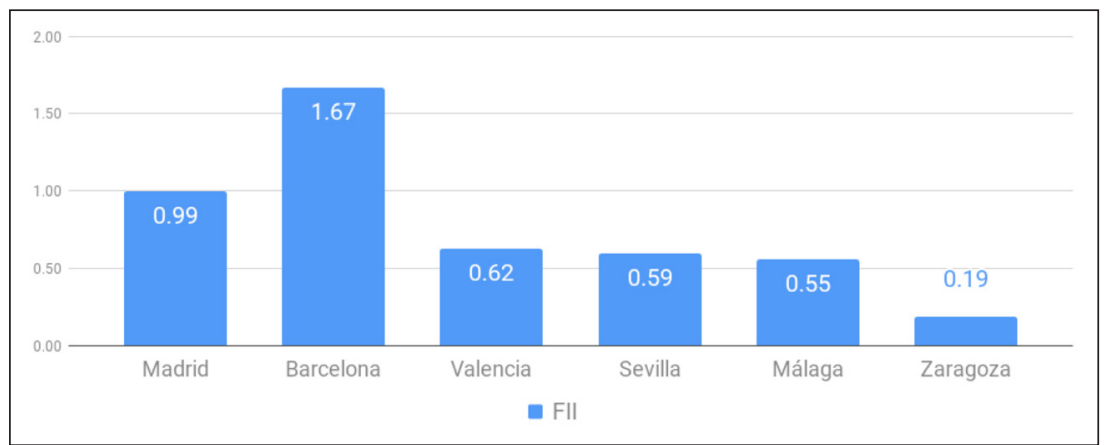

Fuente: Elaboración propia.

\section{Figura 3}

FRECUENCIAS DE LAS PUBLICACIONES CONJUNTAS (2017 Y 2018) OBTENIDAS PARA CADA DESTINO

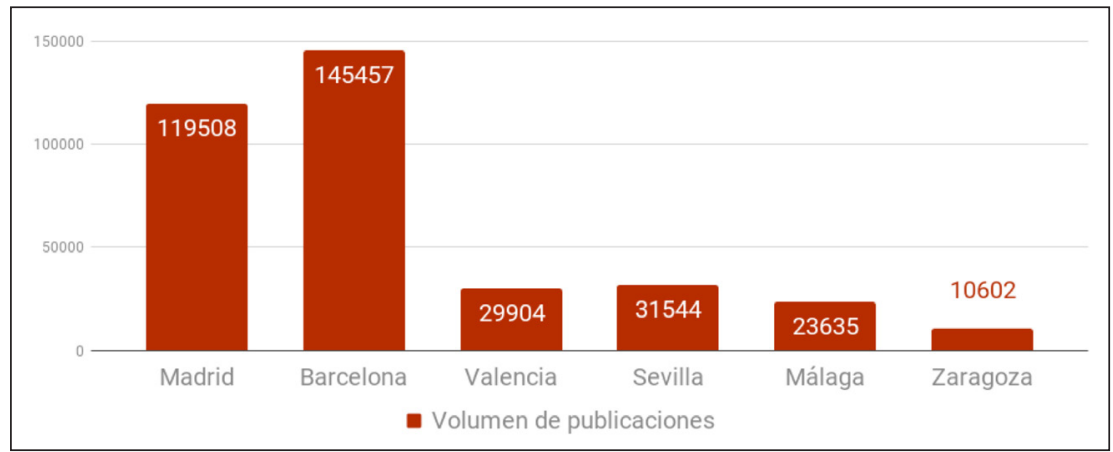

Fuente: Elaboración propia. 
Otro aspecto destacable en la comparativa de la figura 2 y la figura 3 es que mientras Sevilla supera a Valencia ligeramente en número de publicaciones, Valencia supera a Sevilla también mínimamente en el ranking de popularidad de las fotografías y los vídeos (FII). Y ello se debe al efecto de las superiores reacciones de los usuarios, que como se ha visto son las que muestran un mayor éxito de las publicaciones por parte de los usuarios.

Finalmente, cabe destacar que las ciudades pequeñas salen mejor posicionadas en el ranking (figura 2) que si observamos tan solo el número de publicaciones (figura 3), porque el FII, al tener en cuenta el volumen poblacional y las reacciones de los usuarios, esto hace que aumenten sus valores. Por tanto, se destaca la importancia de este índice para medir realmente la popularidad o la imagen de los destinos en Instagram.

\section{CONCLUSIONES}

Este estudio, que analiza la popularidad de los destinos a través de las fotografías y vídeos publicados por los usuarios en Instagram, ha mostrado la importancia de tener en cuenta el volumen poblacional de estos territorios en la medida de su popularidad a través de las publicaciones conseguidas, ya que por lo general los destinos más grandes y con mayor número de habitantes son los que suelen conseguir más publicaciones. Con todo, este estudio también ha mostrado que algunos destinos, con menor número de habitantes que otros, pueden conseguir más publicaciones de fotografías y vídeos, como es el caso de Barcelona o de Sevilla. Ello indica que son destinos más atractivos para las publicaciones, que poseen un mayor número de turistas o visitantes y que además estos usuarios son más activos en Instagram.

El estudio también ha evidenciado la importancia de las reacciones de los usuarios a las publicaciones de fotografías y vídeos para la medición de su popularidad, porque son estas reacciones las que realmente muestran el éxito de las publicaciones a través de la expresión de interés, agrado y la participación de los usuarios. Como muchos autores han demostrado anteriormente (Molinillo et al., 2018, Lu, Chi y Liu, 2015; Prayag y Ryan, 2012), las reacciones de los usuarios son importantes para la creación de la imagen de los destinosy además, también han demostrado que mayores reacciones generan superiores impactos en la creación de una imagen positiva de los destinos (Martín-Santamaría, Beerli-Palacio y Nazzareno, 2017). Por todo ello, las reacciones se han analizado y se han tenido en cuenta para la elaboración del FII, mostrando su importancia para evaluar la popularidad y la imagen de los territorios. Ejemplo de ello es Valencia, que a pesar de que Sevilla la supera en número de publicaciones, Valencia consigue más reacciones por parte de los usuarios, tanto en me gusta como en número de comentarios, aumentando la popularidad y la imagen del destino y su posición en el ranking.

Así pues, el estudio demuestra que el Factor de Impacto en Instagram (FII) es un índice mucho más adecuado para medir la popularidad de los destinos a través de las publicaciones de fotografías y vídeos de los usuarios en Instagram que el simple volumen de publicaciones. Además, también es clave para la creación de rankings de popularidad y aplicable a todo tipo de destino. 
Dado que las fotografías y los vídeos de los usuarios en los medios sociales crean imagen para los destinos (Jenkins, 2003; Míguez-González y Huertas, 2015; Trusov, Bucklin y Pauwels, 2009; Lim, Chung y Weaver, 2012) y Instagram también (Fatanti y Suyadnya, 2015; Molinillo et al., 2018); y debido a que el simple hecho de estar presente en las fotografías y vídeos aumenta la popularidad (Çakici, Kuhzady y Benli, 2017), el FII creado en este estudio muestra el posicionamiento de los destinos creado en la mente de los individuos, y ello es relevante y supone una gran aportación para el sector turístico y para los destinos.

La relevancia de este estudio y del FII para el ámbito turístico es doble. Por un lado, se observa que el turismo, el ocio y la atracción de los destinos influye tanto en la publicación de fotografías y vídeos como en las reacciones de los usuarios. Por otro lado, además, este factor (FII) también nos indica los destinos que son más populares y atractivos respecto al resto, y por tanto conseguirán mayor número de visitantes (Molinillo et al., 2018). Como consecuencia, el FII y el Ranking de popularidad resultan de gran interés para las OMDs, ya que les permitirá conocer su posicionamiento respecto al resto de destinos en la mente de los usuarios (Drakulicet al., 2017; Çakici, Kuhzady y Benli, 2017), lo cual es clave para resultar más atractivos y ser más visitados (Molinillo et al., 2018).

El estudio también corrobora que Instagram es un medio social y una herramienta muy potente para crear imagen y popularidad de los destinos y para conseguir un buen posicionamiento en la mente de los usuarios, tal y como otros autores han demostrado anteriormente (Hanan y Putit, 2013; Fatanti y Suyadnya, 2015; Molinillo et al., 2018). Así pues, las OMDs deberían tener presente la medición del impacto de la popularidad de los destinos a través del uso del FII, que es la principal contribución de este estudio.

Sin embargo, a pesar de que el estudio tiene el valor de georreferenciación para analizar todas las publicaciones de los destinos, también posee limitaciones, como la no distinción entre los autores de las publicaciones analizadas, ya sean turistas o residentes; o la limitación del análisis a periodos muy reducidos de tiempo (tan sólo dos semanas) debido a la inmensa cantidad de datos o publicaciones que se obtienen. Si se hubiera conocido el número de turistas de cada destino por periodo analizado, se hubiera podido determinar con mayor exactitud el impacto del turismo en la georreferenciación de fotografías y vídeos y en la creación de la imagen de los destinos. Con todo, este no era el objetivo del actual estudio, que pretendía un análisis de las publicaciones a nivel global.

En investigaciones futuras se tratará de obtener datos de población flotante y de volumen de ocupación turística y se añadirán estos conceptos al algoritmo creado para la medición del impacto delas fotografías y los vídeos generados por los usuarios. Por otro lado, también se analizarán los temas tratados o aparecidos en dichas fotografías y vídeos publicados en Instagram, y se comparará el análisis de las publicaciones en períodos temporales concretos con una investigación de lo que ocurrió en esos destinos durante esas fechas. Así conoceremos qué les lleva a publicar más fotografías o vídeos de los destinos y qué aspectos son más fotografiados o filmados por los usuarios. Finalmente, también se profundizará más en la relación entre variables analizadas a través del método estadístico de la regresión lineal 


\section{REFERENCIAS}

AITKEN, R. y CAMPELO, A. (2011): «The four Rs of place branding», Journal of Marketing Management, vol. 27 (9-10), pp.913-933.

BALOMENOU, N., GARROD, B. y GEORIADOU, A. (2017): «Making sense of tourists' photographs using canonical variate analysis», Tourism Management, $\mathrm{n}^{\circ}$ 61, pp. 173-179.

BLAIN, C., LEVY, S.E. y RITCHIE, J.B. (2005): «Destination branding: Insights and practices from destination management organizations», Journal of Travel Research, vol. 43 (4), pp. 328-338.

BUHALIS, D. y LAW, R. (2008): «Progress in information technology and tourism management: 20 years on and 10 years after the Internet-The state of eTourism research»,Tourism Management, vol. 29 (4), pp. 609-623.

ÇAKICI, A.C.,KUHZADY, S. y BENLI,S. (2017): «An Image Is Worth A Thousand Words: A Pictorial Analysis of Projected Destination Image of Turkey on Instagram». Disponible en:

https://www.researchgate.net/profile/Salar_Kuhzady/publication/320614475_An_ Image_Is_Worth_A_Thousand_Words_A_Pictorial_Analysis_of_Projected_Destination_Image_of_Turkey_on_Instagram/links/59f06894aca272cdc7ca294e/ An-Image-Is-Worth-A-Thousand-Words-A-Pictorial-Analysis-of-Projected-Destination-Image-of-Turkey-on-Instagram.pdf

CHEN, C.-Ch. y LIN, Y.-H. (2012): «Segmenting Mainland Chinese Tourists to Taiwan by Destination Familiarity: a Factor-cluster Approach»,International Journal of Tourism Research, vol. 14 (4), pp. 339-352.

CHO, Y.-H., WANG, Y. y FESENMAIER, D.R. (2002): «Searching for experiences: The web-based virtual tour in tourism marketing», Journal of Travel \& Tourism Marketing, $\mathrm{n}^{\mathrm{o}} 12(4), \mathrm{pp} .1-17$.

CHOI, S., LEHTO, X.Y. y MORRISON, A.M. (2007): «Destination image representation on the web: Content analysis of Macau travel related websites», Tourism Management, vol.28(1), pp. 118-129.

COOMBS, W.T. y HOLLADAY, S. (2011): «An exploration of the effects of victim visual son perceptions and reactions to crisis events», Public Relations Review, vol. 37 (2), pp. $115-120$.

COSGROVE, D. (2005):«Maps, Mapping Modernity: Art and Cartography in the Twentieth Century»,Imago Mundi, vol. 57 (1), pp. 35-54.

CROMPTON, J. (1992): «Structure of vacation destination choice sets», Annals of Tourism Research, vol. 19 (3), pp. 420-434.

DE BRUYN, A. y LILIEN, G.L. (2008): «A multi-stage model of word-of-mouth influence through viral marketing», International Journal of Research in Marketing, vol. 25 (3), pp. 151-163.

DIARIO LAS AMÉRICAS (2017): «Las 10 ciudades más fotografiadas en Instagram en 2017». Disponible en:https://www.diariolasamericas.com/tecnologia/las-10-ciudadesmas-fotografiadas-instagram-2017-n4139267 
DINHOPL, A. y GRETZEL, U. (2015): «Changing Practices/New Technologies: Photos and Videos onVacation», en Tussyadiah, L. e Inversini, A. (Eds.).Information and Communication Technologies in Tourism 2015,pp. 777-788,Vienna, Austria, Springer.

EKINCI, Y., y HOSANY, S. (2006): «Destination personality: An application of brand personality to tourism destinations», Journal of Travel Research, vol. 45 (2), pp. 127-139.

FASOLO B., MISURACA, R., McCLELLAND, G.H y CARDACI, M. (2006): «Animation attracts: the attraction effect in an on-line shopping environment», Psycholy\& Marketing, vol. 23 (10), pp. 799-811.

FATANTI, M.N. y SUYADNYA, W. (2015): «Beyond User Gaze: How Instagram Creates Tourism Destination Brand?», Procedia-Social and Behavioral Sciences, $\mathrm{n}^{\circ} 211$, pp. $1.089-1.095$.

FYALL, A. y GARROD, B. (2005): Tourism Marketing: A Collaborative Approach. Vol. 18. Clevedon: Channel View Publications.

GALLARZA, M. G., SAURA, I. y HAYDÉE, C.G. (2002): «Destination image: Towards a conceptual framework», Annals of Tourism Research, vol. 29 (1), pp. 56-78.

GOVERS, R. y GO, F.M. (2003): «Deconstructing destination image in the information age», Information Technology and Tourism, vol.6 (1), pp. 13-29.

GOVERS, R. y GO, F.M. (2009): Place branding-glocal, physical and virtual identities constructed, imagined or experienced. Basingstoke, Hampshire, UK: Palgrave Macmillan.

HANAN, H. y PUTIT, N. (2014): «Express marketing of tourism destinations using Instagram in social media networking», en Sumarjan et al. (Eds). Hospitality and Tourism. London, Taylor \& Francis Group, pp. 471-474.

HENDRICKSE, J., ARPAN, L.M.,CLAYTON, R.B. y RIGDWAY, J.L. (2017): «Instagram and college women's body image: investigating the roles of appearance-related comparisons and intrasexual competition», Computers in Human Behavior, $\mathrm{n}^{\circ}$ 74, pp. 92-100.

HO, C.-I., LIN, M.-H., CHEN, H.-M. (2012): «Web users' behavioural patterns of tourism information search: From online to offline», Tourism Management, $\mathrm{n}^{\circ} 33$, pp. 1468-1482.

HSIEH, Y.-C. y CHEN, K.-H. (2011): «How different information types affect viewer's attention on internet advertising», Computers in Human Behavior, vol. 27 (2), pp. 935-945.

HUERTAS, A. (2018): «How live videos and stories in social media influence tourist opinions and behavior», Information Technology \&Tourism, vol. 19 (1-4), pp. 1-28.

HUERTAS, A. y MARINÉ-ROIG, E. (2018): «Searching and sharing of information in social networks during the different stages of a trip», Cuadernos de Turismo, $\mathrm{n}^{\circ} 42$, pp.185-212.

HUERTAS, A., MÍGUEZ-GONZÁLEZ, M.I. y LOZANO-MONTERRUBIO, N. (2017): «YouTube usage by Spanish tourist destinations as a tool to communicate their identities and brands», Journal of Brand Management, vol. 24 (3), pp. 211-229.

HUERTAS, A., SETÓ-PÀMIES, D. y MÍGUEZ-GONZÁLEZ, M.I. (2015): «Comunicación de destinos turísticos a través de los medios sociales», El profesional de la Información, vol. 24 (1), pp.15-21. 
IBRAHIM, E.E. y GILL, J. (2005): «A positioning strategy for a tourist destination, based on analysis of customers' perceptions and satisfactions», Marketing Intelligence \& Planning, vol. 23 (2), pp. 172-188.

JABREEL, M., HUERTAS, A., y MORENO, A. (2018): «Semantic analysis and the evolution towards participative branding: Do locals communicate the same destination brand values as DMOs? », PloS one, vol. 13 (11), pp. e0206572.

JACOBSEN, J. K.S. y MUNAR, A.M. (2012): «Tourist information search and destination choice in a digital age», Tourism Management Perspectives, vol. 1 (1), pp. 39-47.

JANSSON, A. (2002): «Spatial phantasmagoria: the mediatization of tourism experience», European Journal of Communication, vol. 17 (4), pp. 429-443.

JENKINS, O. (2003): «Photography and travel brochures: the circle of representation», Tourism Geographies, vol. 5 (3), pp. 305-28.

KAVARATZIS, M. (2012): «From “necessary evil” to necessity: Stakeholders' involvement in place branding», Journal of Place Management and Development, vol. 5 (1), pp. 7-19.

KIM, S.-B., KIM, D.-Y. y BOLLS, P. (2014): «Tourist mental-imagery processing: Attention and arousal», Annals of Tourism Research, $\mathrm{n}^{\circ}$ 45, pp. 63-76.

KHLAT, M. (2014): Tourism, the most popular sector on Instagram. (Online). Disponible en: https://www.tourmag.com/Tourism-the-most-popular-sector-on-Instagram_a65405. htmlAccedido: enero 2018

KOERNIG, S.K. (2003): «E-scapes: The electronic physical environment and service tangibility», Psychology \& Marketing, vol. 20 (2). pp. 151-167.

KOVACEVIC, N.D., KOVACEVIC, L., STANKOV, U., DRAGICEVIC, V y MILETIC, A. (2017): «Applying destination competitiveness model to strategic tourism development of small destinations: The case of South Banat district». Journal of Destination Marketing \& Management, vol. 8, pp. 114-124.

KUHZADY, S., GHASEMI, V. y HASHEMI, S. (2017): «Pictorial analysis of the projected destination image: Portugal on Instagram», en $7^{\text {th }}$ Advances in Tourism Marketing Conference. Proceedings Book. pp. 214-222.

LALICIC, L., HUERTAS, A., MORENO, A., y JABREEL, M. (2018): «Which emotional brand values do my followers want to hear about? An investigation of popular European tourist destinations», Information Technology \& Tourism, vol. 21 (1), pp. 63-81.

LÁZARO, M. (2017): «Del 20 al 1: las ciudades españolas más fotografiadas en Instagram». Tendencias. Disponible en: https:/www.huffingtonpost.es/2017/08/29/del20-al-1-las-ciudades-espanolas-mas-fotografiadas-en-instagram_a_23189143/

LEE, W. y GRETZEL, U. (2012): «Designing persuasive destination websites: a mental imagery processing perspective», Tourism Management, 33 (5), pp. 1270-1280.

LIM, Y., CHUNG, Y. y WEAVER, P.A. (2012): «The impact of social media on destination branding consumer-generated videos versus destination marketer-generated videos», Journal of Vacation Marketing, vol. 18 (3), pp. 197-206.

LLODRÀ-RIERA, I., MARTÍNEZ-RUIZ, M.P., JIMÉNEZ-ZARCO, A.I. e IZQUIERDOYUSTA, A. (2015): «A multidimensional analysis of the information sources construct and its relevance for destination image formation», Tourism Management, $\mathrm{n}^{\circ}$ 48, $\mathrm{pp}$. 319-328. 
MACK, R. W., BLOSE, J.E. y PAN, B. (2008): «Believe it or not: Credibility of blogs in tourism», Journal of Vacation Marketing, vol. 14 (2), pp. 133-44.

MARIANI, M.M., DI FELICE, M. y MURA, M. (2016): «Facebook as a destination marketing tool: Evidence from Italian regional Destination Management Organizations», Tourism Management, $\mathrm{n}^{\circ}$ 54, pp. 321-343.

MARKWELL, K.W. (1997): «Dimensions of photography in a nature-based tour», Annals of Tourism Research, vol. 24 (1), pp. 131-55.

MÍGUEZ-GONZÁLEZ, M.I. y HUERTAS, A. (2015): «The power of photographs in the communication and public relations of tourist destinations and their brands through Facebook and Flickr», Catalan Journal of Communication \& Cultural Studies, vol. 7 (2), pp. 197-215.

MOLINILLO, S., LIÉBANA-CABANILLAS, F., ANAYA-SÁNCHEZ, R. y BUHALIS, D. (2018): «DMO online platforms: Imageandintention to visit»,Tourism Management, n65,pp. 116-130.

MORGAN, N. y PRITCHARD, A. (1998): Tourism promotion and power: Creating images, creating identities. Chichester (UK): John Wiley \& Sons Ltd.

MORGAN, N.J., PRITCHARD, A., y PIGGOTT, R. (2003): «Destination branding and the role of the stakeholders: The case of New Zealand», Journal of Vacation Marketing, vol. 9 (3), pp. 285-299.

MORGAN, N., PRITCHARD, A. y PRIDE, R. (2007): Destination branding. Routledge. MULL, I.R. y LEE, S.-E. (2014): «PIN pointing the motivational dimensions behind Pinterest», Computers in Human Behavior, n 33, pp. 192-200.

ORDÓÑEZ DE PABLOS, P. y AUNG, Z.M. (2017): Tourism and Opportunities for Economic Development in Asia.

PANIAGUA, F. y HUERTAS, A. (2018): «El contenido en los medios sociales de los destinos turísticos y la búsqueda de información de los usuarios», Cuadernos de Turismo, $\mathrm{n}^{\mathrm{o}} 41$, pp. 513-534.

PAPATHANASSIS, A. y KNOLLE, F. (2011): «Exploring the adoption and processing of online holiday reviews: a grounded theory approach», Tourism Management, vol. 32 (2), pp. 215-224.

PEARCE, P.L., WU, M.-Y. y CHEN, T. (2015): «The spectacular and the mundane: Chinese tourists' online representations of an iconic landscape journey», Journal of Destination Marketing \& Management, vol. 4 (1), pp. 24-35.

PIKE, S. (2009): «Destination brand positions of a competitive set of near-home destinations», Tourism Management, vol. 30 (6), pp. 857-866.

PIKE, S. (2012): «Destination positioning opportunities using personal values: Elicited through the Repertory Test with Laddering Analysis», Tourism Management, vol. 33 (1), pp. 100-107.

PIKE, S. (2017): «Destination positioning and temporality: Tracking relative strengths and weaknesses over time», Journal of Hospitality and Tourism Management, $\mathrm{n}^{\circ} 31$, pp. 126-133.

PIKE, S., y PAGE, S.J. (2014): «Destination Marketing Organizations and destination marketing: A narrative analysis of the literature», Tourism Management, $\mathrm{n}^{\circ}$ 41, pp. 202-227. 
QU, H., KIM, L.H. e IM, H.H.(2011): «A model of destination branding: Integrating the concepts of the branding and destination image», Tourism Management, vol. 32 (3), pp. 465-476.

SCHWAIGHOFER, V. (2013): Tourist Destination Images and Local Culture: Using the Example of the United Arab Emirates. Salsburg: Springer Science \& Business Media.

SEABRA, C., ABRANTES, J.L. y LAGES, L.F. (2007): «The impact of using nonmedia information sources on the future use of mass media information sources: The mediating role of expectations fulfillment», Tourism Management, vol. 28 (6), pp. 1.541-1.554.

STEPCHENKOVA, S. y ZHAN, F. (2013): «Visual destination images of Peru: Comparative content analysis of DMO and user-generated photography», Tourism Management, $\mathrm{n}^{\circ} 36$, pp. 590-601.

TASCI, A.D.A. (2008): «Social Distance: The Missing Link in the Loop of Movies, Destination Image, and Tourist Behavior? »,Journal of Travel Research, vol. 47 (4), pp. 494-507.

TASCI, A.D.A. y GARTNER, W.C. (2007): «Destination Image and Its Functional Relationships», Journal of Travel Research, vol. 45 (4), pp. 413-425.

THAM, A., CROY, G. y MAIR, J. (2013): «Social media in destination choice: Distinctive electronic word-of-mouth dimensions», Journal of Travel \& Tourism Marketing, vol. 30 (1-2), pp. 144-155.

TRECEBITS (2017): Las ciudades más fotografiadas en Instagram. Accesibleen:https:// www.trecebits.com/2017/01/11/las-ciudades-mas-fotografiadas-en-instagram/

TRUSOV, M., BUCKLIN, R.E. y PAUWELS, K. (2009): «Effects of word-of-mouth versus traditional marketing: findings from an internet social networking site», Journal of Marketing, vol. 73 (5), pp. 90-102.

TUSSYADIAH, L.P. y FESENMAIER, D.R. (2009): «Mediating Tourist Experiences: Access to Places via Shared Videos», Annals of Tourism Research, vol. 36 (1), pp. 24-40.

VERMEULEN, I.E. y SEEGERS, D. (2009): «Tried and tested: The impact of online hotel reviews on consumer consideration»,Tourism Management, vol. 30 (1), pp. 123-127.

WILLIAMS, S. (2015): «Here Now! Social media and the psychological city», en Inscribing a Square Urban Data as Public Space, Springer, pp. 88-92.

XIANG, Z. y GRETZEL, U. (2010): «Role of social media in online travel information search», Tourism Management, vol. 31 (2), pp. 179-88.

ZILLMAN, D., GIBSON, R. y SARGENT, S.L. (1999): «Effects of photographs I newsmagazine reports on issue perception», Media Psychology, vol. 1 (3), pp. 207-228. 